\title{
Performance Analysis of Feedback Type WDM Optical Routers under Asynchronous and Variable Packet Length Self-Similar Traffic
}

\author{
Shou-Kuo Shao, Meng-Guang Tsai, Hen-Wai Tsao, Paruvelli Sreedevi, and Jingshown Wu, Senior Member, IEEE
}

\begin{abstract}
In this paper, we investigate the assessment of packet loss and system dimensioning of Feedback (FB) type Wavelength Division Multiplexing (WDM) optical routers under self-similar IP traffic. We first study the packet loss performance for two types of WDM optical routers with asynchronous and variable packet length. Based on the simulation results, we proved that a 16x16 FB type WDM optical IP router employing more than 4 re-circulated ports without using void filling algorithm can provide better performance. We then present the system dimensioning issues of FB type WDM optical routers, by showing that the performance of $\mathrm{FB}$ type routers is a function of number of re-circulated ports (re-circulated wavelengths), buffer depth, re-circulation limits and traffic characteristics. Hence, in order to be effective, the FB type routers must be dimensioned with appropriate number of re-circulated ports, re-circulation limits and optimal delay line unit under relevant traffic characteristics to achieve high switching performance.
\end{abstract}

Index Terms-Asynchronous, feedback type, self-similar traffic, variable packet length, WDM optical router.

\section{INTRODUCTION}

$\mathbf{T}$ HE explosive growth of Internet traffic demands more bandwidth on the networks. While IP backbone systems are being realized using Synchronous Optical Network / Synchronous Digital Hierarchy (SONET/SDH) and Asynchronous Transfer Mode (ATM) technologies, existing transport networks will not yield the bandwidth-effective transport and routing schemes for IP traffic. The integration of IP and Dense WDM (DWDM) has been the most popular research and development area for next generation Internet. The ultimate WDM optical routers will combine the advantage of DWDM with all opitcal packet switching capabilities for IP centric services [1].

IP packets are born to be asynchronous and variable packet length. Hence, the transfer mode based on variable length packets and asynchronous operation is more effective [2]. For Internet and IP traffic properties, recent measurements

Manuscript received October 15, 2003. This work was supported in part by Ministry of Education Program for Promoting Academic Excellence of Universities and National Science Council, Taiwan, R.O.C. under Grant 89EFA06-2-4-7 and Grant NSC 91-2213-E-002-106.

Shou-Kuo Shao, Meng-Guang Tsai, Hen-Wai Tsao, Paruvelli Sreedevi and Jingshown Wu are with the Department of Electrical Engineering and Graduate Institute of Communication Engineering, National Taiwan University, Taipei, Taiwan 106, R.O.C. (phone: 886-2-23635251 ext.:519; fax:886-223638247; e-mail: wujsh@cc.ee.ntu.edu.tw)

Shou-Kuo Shao is also with the Network Operation Supporting Technology Lab., Chunghwa Telecommunication Laboratories, Chunghwa Telecom Co., Ltd., Yang-Mei, Taoyuan 326, Taiwan. (e-mail: skshao@cht.com.tw) reported by many researchers have shown that traffic in both Ethernet and WAN networks tends to be bursty in many or all time scales [3], [4]. This bursty traffic can be characterized mathematically as self-similar or long-range dependent (LRD) traffic. Research results have shown that LRD) traffic has severe impact on switch performance and network design. Therefore, it is a must to investigate the performance of WDM optical routers under asynchronous and variable packet length selfsimilar traffic.

There are two different types of WDM optical routers: one is feed-forward (FF) type [5], which employs output queueing buffer only, and the other is FE type or the socalled partially shared buffering (PSB) type, which implements a common shared re-circulated buffer to extend the buffer depth [6], [7]. The FF type WDM optical routers employed with wavelength conversion work well under both Bernoulli and short range dependent (SRD) traffic because multiserver queues could interleave the packets among the available wavelengths. Wavelength conversion applied to optical routers is a very effective way of improving the performance [8]. This is even more important in view of the argument that the buffer depth of the fiber delay line buffers is likely to be small. Providing massive multiserver queues is also one of the most effective strategies available for combating the effects of self-similar traffic [2]. However, in the case of limited range of wavelength conversions, we find that the performance of the FF type optical routers cannot be improved significantly under self-similar traffic [9]. Recent efforts have concentrated on simplifying the optical switch matrix away from the concept of fully shared optical buffers and more towards the concept of partially shared buffers [7]. FB type WDM optical routers employed with wavelength conversion could provide multiserver queues with larger buffer depth as well as greater traffic smoothing capability. Hence it can improve the packet loss performance and reduce the system complexity [6], [7].

When WDM optical routers operate under asynchronous and variable packet length mode, there will be voids induced in the fiber delay lines [1], [2]. These voids rnake the channels idle and are unusable for storing newly arriving packets. Thus, the performance in terms of probability of packet loss (PPL) is inferior to the routers operating under synchronous and fixed packet length mode. Void filling (VF) algorithm has been proposed to alleviate this problem. However, its process is too complicated and computation-intensive to be realized 
in high-speed optical networks. We have shown that FB type WDM optical routers employing wavelength conversion can provide superior performance over FF type routers in various contention resolution schemes [10]. Based on our results, we have proved that a $16 \times 16$ FB type WDM optical router employing more than 4 re-circulated ports without using void filling algorithm can provide better performance than that of FF type routers using void filling algorithm under asynchronous and variable packet length self-similar traffic with Hurst parameter, $\mathrm{H}=0.9$ and traffic load $=0.8$.

In this paper, we investigate the assessment of packet loss and system dimensioning of FB type WDM optical routers under self-similar IP traffic. We first review the architecture of FB type WDM optical routers in Section II. We present that effective FB type WDM optical routers must be dimensioned with appropriate number of re-circulated ports, re-circulation limits and optimal delay line unit under relevant traffic characteristics to achieve high performance in Section III. Finally we conclude this paper in Section IV.

\section{ARCHITECTURE OF FB TYPE WDM OPTICAL ROUTERS}

A general block diagram of FB type WDM optical routers is illustrated in Fig. 1(a) [6], [7]. For an N x N FB type WDM optical routers with $n$ wavelengths per port, there are $\mathbf{R}$ $(1 \leq R \leq N)$ re-circulated (feedback) ports to re-circulate packets that cannot be switched out to their destinations (primary output buffers) back to the input end of the router. Those recirculated packets from all input ports commonly share the recirculated buffers. Fig. 1(b) shows an FB type WDM optical router implemented in broadcast-and-select architecture. From this, we could observe that the architecture of FF type routers is indicated above dashed line [5]. By employing wavelength conversion, the required number of fiber delay lines could be minimized. The number of re-circulated wavelengths is $W$, where $W$ is equal to the number of wavelengths per port, $n$, times the number of re-circulated ports, R. Hence, $W$ and $R$ are equivalent in dimensioning FB type WDM optical routers.

Contention occurs, when packets are switched to the same output port at the same time. Those packets that can not be scheduled into their primary output buffers are stored in the common shared re-circulated buffers. These backlogged packets are kept re-circulating in the common shared buffers until they get the chance to be switched out. However, practically, while these packets re-circulate in the common shared buffers, optical components such as wavelength converters, and optical amplifier would generate an accumulated Amplified Spontaneous Emission (ASE) noise to hinder packet re-circulations in the buffers due to signal to noise ratio degradation [11]. This accumulated noise limits the number of re-circulations in the re-circulated buffers. For FB type WDM optical routers, this effect could be described by a system parameter, re-circulation limit, b. A packet that has re-circulated $b$ times in the buffers and is not able to enter its desired output port yet, will be dropped by the routers.

Table I shows the meaning of system parameters and the corresponding symbols that are used in this paper for dimensioning the FB type WDM optical routers.

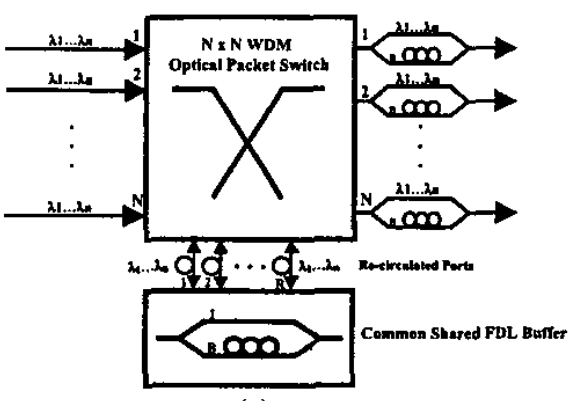

(a)

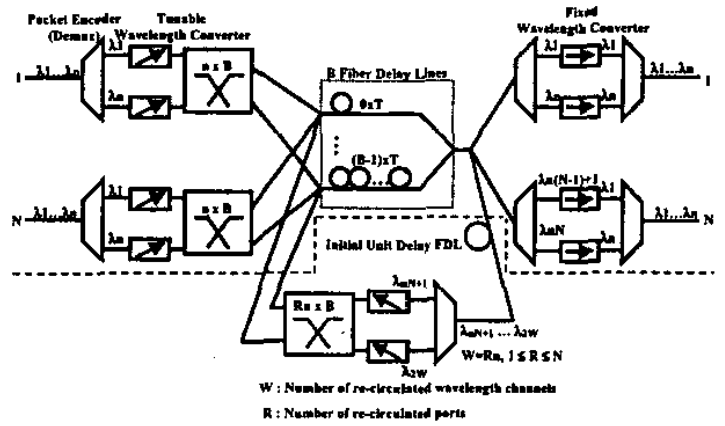

(b)

Fig. 1. (a) Typical block diagram of FB type WDM optical routers; (b) Broadcast-and-select architecture of FB type WDM optical routers.

TABLE I

SYSTEM PARAMETERS OF FB TYPE WDM OPTICAL ROUTERS.

\begin{tabular}{||c|c||}
\hline Symbol & Meaning of Parameter \\
\hline $\mathrm{N}$ & Number of $\mathrm{I} / \mathrm{O}$ ports \\
\hline $\mathrm{n}$ & Number of wavelengths per port \\
\hline $\mathrm{R}$ & Number of re-circulated ports \\
\hline $\mathrm{W}$ & Number of re-circulated wavelengths \\
\hline $\mathrm{b}$ & Re-circulation limit \\
\hline $\mathrm{B}$ & Buffer depth (number of fiber delay lines) \\
\hline DLU & Fiber delay line unit \\
\hline
\end{tabular}

\section{PERFORMANCE ANALYSIS - SIMULATIONS RESULTS}

We first investigate the packet loss performance of the FB type WDM optical routers under asynchronous, variable packet length self-similar traffic. We have done programming in an ad-hoc discrete event simulator (DES) using raw $\mathrm{C}++$ code to perform the analysis. In all the following simulations, the number of $\mathrm{V} / \mathrm{O}$ ports, $\mathrm{N}$, and the number of wavelength per port, $n$, are 16. The packet destinations are uniformly distributed among all the output ports. We use an aggregated $\mathrm{ON}-\mathrm{OFF}$ process modulated with a Pareto distribution to simulate asynchronous, variable packet length self-similar traffic [2], [13], [14]. The Pareto distribution with cumulative distribution function (CDF), $P[X<x]=1-(\theta / x)^{\alpha}$, for $\alpha>0$, has the degree of self-similarity, $\mathrm{H}=(3-\alpha) / 2$, where $\mathrm{H} \in(0.5,1)$ and $\alpha \in(1,2)$. ON periods represent bursts of packets and $\mathrm{OFF}$ periods denote the interarrival times. The location parameter of the ON time Pareto distribution, $\theta$, is 
set to be 400 bytes for comparison [2]. The switching scenario is burst switching (without considering signaling and control protocols), i.e., the whole burst is switched as a single entity. For routers employing void filling algorithm, the electronic memory space for recording voids is set to be 40 fields [2].

\section{A. Packet Loss Assessment}

Since the packet loss performance of WDM optical routers under asynchronous and variable packet length traffic is dependent upon both the traffic and router parameters, we illustrate the performance in "spectral dependence" plot (i.e., a plot that shows the dependence of the probability of packet loss on the length of delay line unit [2]) for 16×16 WDM optical routers with buffer depth, $B=10$ in Figs. 2(a) and 2(b).

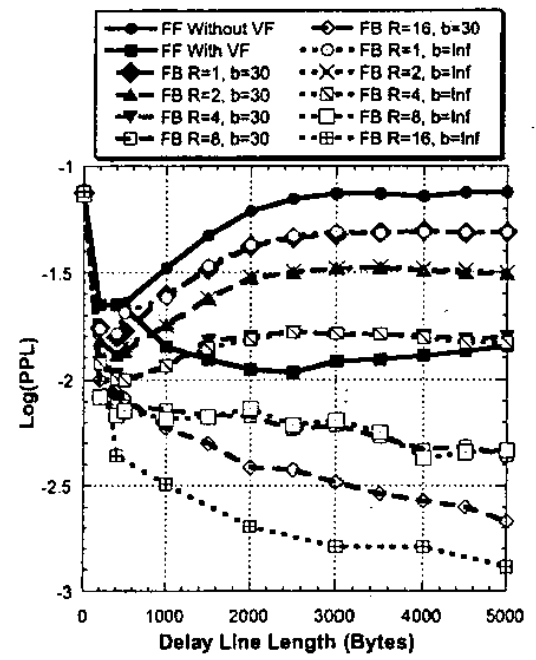

(a)

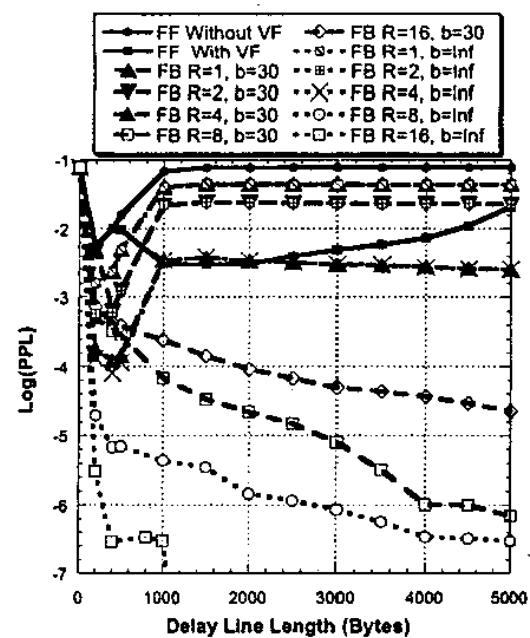

(b)

Fig. 2. Spectral dependence plots of the probability of packet loss of $16 \times 16$ FB type routers without void filling and FF type with and without void filling. (a) $\mathrm{B}=10, \mathrm{H}=0.9$ and traffic load $=0.8 ;(\mathrm{b}) \mathrm{B}=10, \mathrm{H}=0.7$ and traffic load $=0.8$.

From Figs. 2(a) and 2(b), we observe that: 1) Voids caused by asynchronous and variable length packets can degrade the performance. Void filling algorithm does improve the packet loss performance, however, it is very computation-intensive, 2) FB type routers with $R>4$ and $b=$ infinite, without using void filling is better than FF type with void filling algorithm. The result remains the same when $b$ is degraded by noise constraints to 30 . As illustrated in the figures, when $R \leq 8$, $\mathrm{b} \geq 30, \mathrm{H}=0.9$ and traffic load $=0.8$, re-circulation limit has no effect upon the packet loss performance. When $R \leq 4$, $\mathrm{b} \geq 30, \mathrm{H}=0.7$ and traffic load $=0.8$, the results are the same. The packet loss performance of the case $b=30$ and the case $\mathrm{b}=$ infinite becomes distinct, when $\mathrm{R}=16$ at $\mathrm{H}=0.9$ and when $\mathrm{R} \geq 8$ at $\mathrm{H}=0.7$. Notice that the packet loss performance of $R=8$ is better than $R=16$ under $H=0.7$ and $b=30$, however, when $H=0.7$ and $b=$ infinite, the performance of WDM optical routers with $R=16$ is better.

Fig. 3 shows the comparisons between 16x16 FF type routers with and without using void filling algorithm, and $16 \times 16$ FB type routers with constraint of re-circulation limit, $\mathrm{b}=30$, without using void filling algorithm, for both with and without using multipath routing (MPR) nodes [15]. The buffer depth, $\mathrm{B}$, is 10 and the input self-similar traffic has a Hurst parameter, $\mathrm{H}=0.9$ and traffic load $=0.8$. The packet loss performance of FF type routers with optimal delay line length is slightly better than that of FB type routers with $R=16$ for using MPR nodes. This means that the residual bandwidth of FB type routers is smaller. Thus the buffer utilization for each output port is higher for FB type routers.

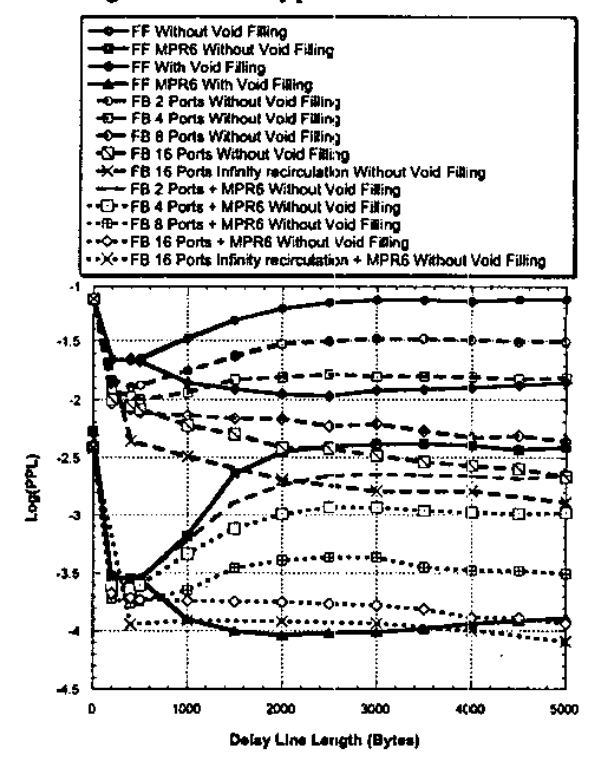

Fig. 3. Spectral dependence plot of the probability of packet loss of $16 \times 16 \mathrm{FB}$ type routers without void filling algorithm and $16 \times 16$ FF type routers with and without void filling algorithm, for both with and without using MPR nodes, when $B=10, b=30$ at $H=0.9$ and traffic load $=0.8$.

All of the above results show that packet loss of FB type WDM optical routers under asynchronous and variable packet length self-similar traffic is due to five factors: (1) availability of buffer depth, (2) feedback bandwidth, (3) re-circulation limits due to noise constraints, (4) delay line length unit (DLU) and (5) traffic characteristics.

To investigate this, we then depict the packet loss performance of FF and FB type routers with optimal delay line 
length (chosen from Figs. 2(a) and 2(b)) when $B=10$ and $b=30$ at various $H$ in Fig. 4. Based on these results, we confirm that $16 \times 16$ FB type routers with $R>4$, without using void filling algorithm are indeed superior to $16 \times 16 \mathrm{FF}$ type routers with void filling algorithm under various $H$. Notice that the performance of FB type routers with $R=16$ is inferior to $F B$ type with $R=8$ under $b=30$ and moderate $\mathrm{H}$, as illustrated in Fig. 4. This reveals that the performance of FB type router under asynchronous and variable packet length self-similar traffic is indeed a function of $R$ (i.e., W), B, b, DLU and traffic parameters. Hence, in order to be effective, the $\mathrm{FB}$ type routers must be dimensioned with appropriate number of re-circulated ports and traffic characteristics with considerations of re-circulation limit and optimal delay line unit.

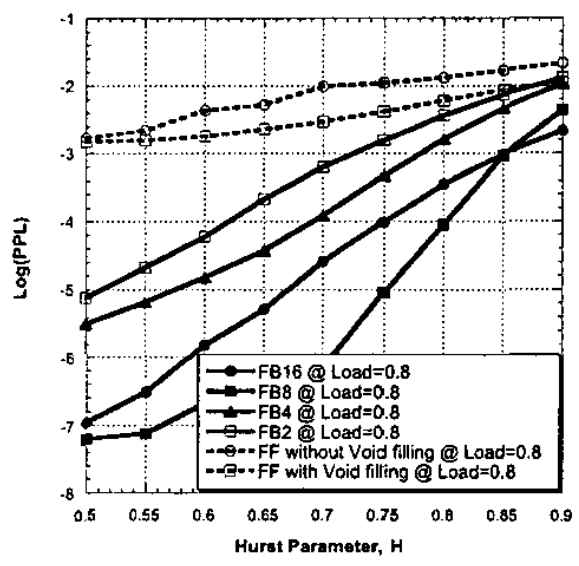

Fig. 4. Packet loss of $16 \times 16$ FB type vs. FF type routers with optimal delay line length when $B=10$ and $b=30$ at varying $H$.

\section{B. System Dimensioning lssues}

To understand the effectiveness of FB type WDM optical routers dimensioned with appropriate number of re-circulated ports, re-circulation limit and optimal delay line unit under relevant traffic characteristics, we depict the system dimensioning issues of FB type WDM optical routers under asynchronous and variable packet length self-similar traffic in the following figures.

Fig. 5 depicts the packet loss performance of $16 \times 16 \mathrm{FB}$ type WDM optical routers with $R=16$ and $D L U=5000$ bytes, as a function of re-circulation limits, $b$, at various $B$, under $\mathrm{H}=0.9$ and traffic load $=0.8$. From this, we observe: 1) The decreasing rates of packet loss probability are much less than the rates of exponential decays (actually they are polynomial decays). Hence increasing the buffer depth could only provide a little help on the switching performance. 2) There would be a saturation floor for the packet loss performance as the recirculation limits increase. This means that employing perfect components with extreme low noise won't be necessary for FB type WDM optical routers with buffer depth only a few tens and under heavier self-similar traffic.

On the other hand, the packet loss performance of FB type WDM optical routers employing with two different values of

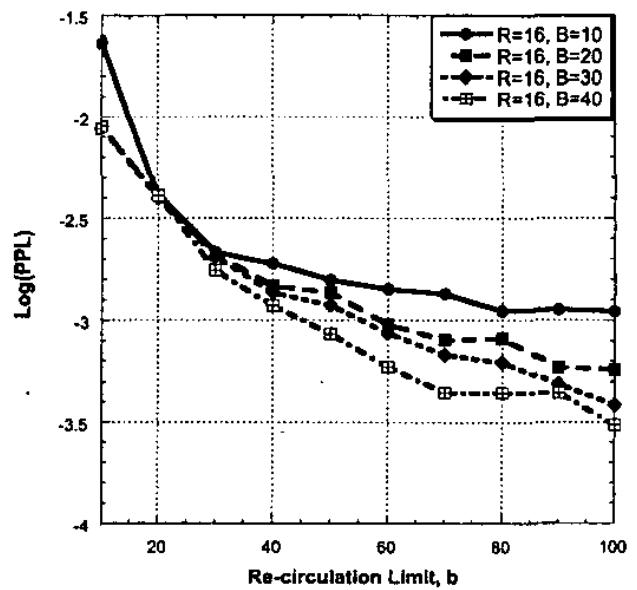

Fig. 5. Packet loss performance of FB type WDM optical routers with $R=16$ and $D L U=5000$ bytes, as a function of re-circulation limits, b, at various $B$, under $H=0.9$ and traffic load $=0.8$.

DLU as a function of re-circulation ports, $R$, at $B=10, H=$ 0.9 and traffic load $=0.8$ is illustrated in Fig. 6 . Fig. 6 shows the system dimensioning of $16 \times 16$ FB type WDM optical routers under higher degree asynchronous and variable packet length self-similar traffic. It is obvious from Fig. 6 that we don't have to employ full bandwidth for storing and re-circulating packets in the common shared buffers. Thus the system complexity of FB type WDM optical routers could be reduced and still retain the satisfactory performance.

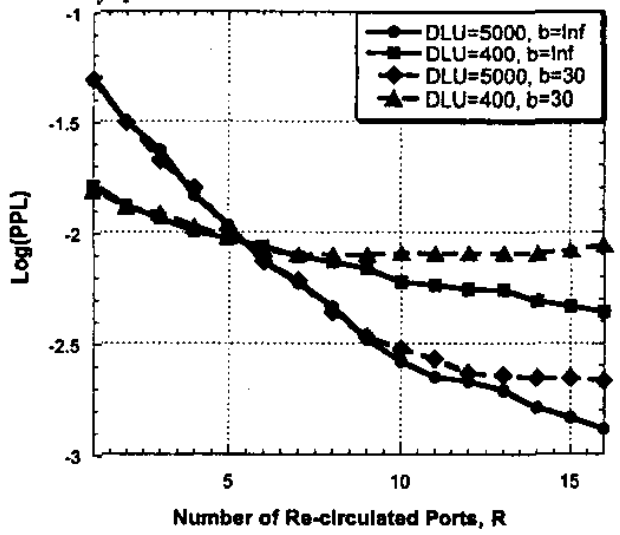

Fig. 6. Packet loss of $16 \times 16$ FB type WDM optical routers as a function of re-circulated ports, at $\mathrm{B}=10, \mathrm{H}=0.9$ and traffic load $=0.8$.

Finally in order to study the effect of buffer depth, B, and the number of re-circulated ports, $R$ (i.e., number of feedback wavelengths, W) upon the packet loss performance, we sketch the packet loss performance as a function of $B$ and $R$ with the constraint $B \times R=16$ under $H=0.9$ and traffic load $=0.8$ in Figs. 7(a) and 7(b). The values of optimal DLU are chosen (from Fig. 2(a)) for each 16x16 FB type WDM optical routers with different number of re-circulated ports. Fig. 7(a) is the case that re-circulation limit, $b=$ infinite and Fig. 7(b) is the case that re-circulation limit, b, is with noise constraint and equal to 30. Figs. 7(a) and 7(b) show packet loss performance trade-offs of buffer depth versus number of re-circulated ports (re-circulated wavelengths). Notice that the 
packet loss performance of routers employing larger buffer depth and fewer re-circulated ports are inferior to the routers employing greater number of re-circulated ports and with smaller buffer depth. This means that the number of re circulated ports in wavelength domain plays a major role compared to the buffer depth in time domain for contention resolution. Hence, in order to be effective, the FB type WDM optical routers must be dimensioned with a correct coupling of number of buffer depth and re-circulated ports.
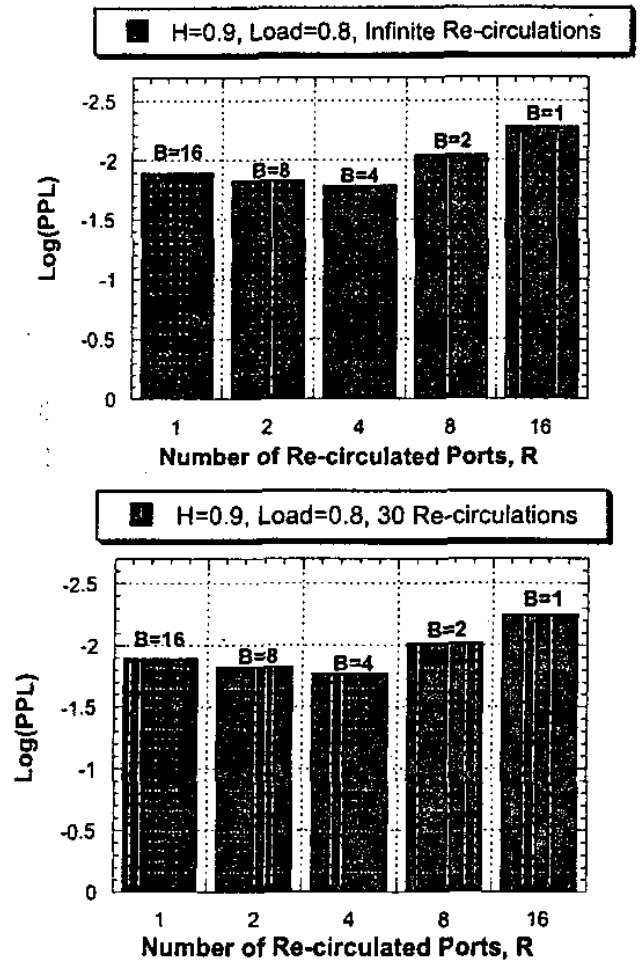

(b)

Fig. 7. Packet Loss at $\mathrm{H}=0.9$ and traffic load $=0.8$ of different choices of the optical buffer resource for $16 \times 16$ WDM optical routers, under the constraint $B \times R=16$. (a) $b=$ infinite re-circulations; (b) $b=30$ re-circulations.

\section{CONCLUSIONS}

Self-similar traffic has severe impact on the switching performance. Voids caused by asynchronous and variable length packets can degrade the performance further. Void filling algorithm could improve the switching performance, however, it is computation-intensive. FF type routers with wavelength conversion work well under both Bernoulli and bursty traffic. However, the performance of $\mathrm{FF}$ type routers cannot be significantly improved under self-similar traffic, in the case of limited number of wavelength channels. FB type routers employing wavelength conversion could provide larger buffer depth as well as greater traffic smoothing capability.

We have studied the performance of these types of WDM optical routers under asynchronous and variable packet length self-similar traffic. The FB type WDM optical routers are superior to the FF type WDM optical routers under selfsimilar traffic, because the entire buffers are shared commonly, and are not exclusively arranged to each output port. The packets are stored and re-circulated in the common shared buffers. However, practically, accumulated ASE noise caused by wavelength converters and optical amplifiers could hinder packet re-circulations in the buffers.

We have shown that the performance of FB type router under asynchronous and variable packet length self-similar traffic is a function of number of re-circulated ports, $R$ (i.e., number of re-circulated wavelength, W), buffer depth, B, recirculation limit, $b$, fiber delay line length unit, DLU and traffic parameters. Hence, in orcler to be effective, the FB type routers must be dimensioned with appropriate number of re-circulated ports, re-circulation limit and optimal delay line unit under relevant traffic characteristics. Based on the results, we conclude that $16 \times 16 \mathrm{FB}$ type WDM optical routers with $\mathrm{R}>4$, without using void filling algorithm can provide better performance under self-similar IP traffic.

\section{REFERENCES}

[1] Franco Callegati, Giorgio Corazza and Carla Raflaelli, "Exploitation of DWDM for optical packet switching with quality of service guarantees," IEEE J. Select. Areas Commun., vol. 20, pp. 190-201, Jan. 2002.

[2] Lubo Tancevski, Siva Yegnanarayanan, Gerardo Castanon, Lakshman Tamil, Francesco Masetti and Tom McDermott, "Optical Routing of Asynchronous, Variable length packets," IEEE J. Select. Areas Commun. , vol. 18, pp. 2084-2093, Oct. 2000.

[3] W. E. Leland, M. S. Taqqu, W. Willinger and D. V. Wilson, "On the self-similar nature of Ethemet traffic (extended version)," IEEE/ACM Trans. Nerworking, vol. 2, no. 1, pp. 1-15, Feb. 1994

[4] M. E. Crovella and A. Bestavros, "Self-similarity in World Wide Web traffic: Evidence and possible causes," IEEE/ACM Trans. Nerworking, vol. 5 , no. 6, pp. $835-846$, Dec. $199 \%$

[5] Soeren L. Danielsen, B. Mikkelsen, C. Joergerssen, T. Durhuus, K. Stubkjaer, "WDM packet switch architecture and analysis of the influence of tunable wavelength converters on the performance", IEEE/OSA J. Lightwave Techno., vol. 15, pp. 219-227, Feb. 1997.

[6] Juan Diao and Pak. L. Chu, "Analysis of partially shared buffering for WDM optical packet switching," IEEE/OSA J. Lightwave Technol., vol. 17, no. 12, pp. 2461-2469, Dec. 1999.

[7] Lubo Tancevski, An Ge and Gerardo Castnon, "Optical packet switchi with partially shared buffers: Design Principles," in Proc. OFC '2001. Anaheim, Mar. 2001, paper TuK3.

[8] Soeren L. Danielsen, Peter B. Hansen and Kristian E. Stubkjaer, "Wavelength conversion in optical packet switching," IEEE/OSA J. Lightwave Technol., vol. 11, no. 12, pp. 2095-21.08, Dec. 1998.

[9] G. Shen, S. K. Bose, T. H. Cheng, C. Lu, T. Y. Chai, "Performance Study on a WDM packet switch with limited-range wavelength converters", IEEE Commun. Letters, vol. 5, no. 10, pp. 432-434, Oct. 2001.

[10] Shou-Kuo Shao, Meng-Guang Tsai, Hen-Wai Tsao, Paruvelli Sreedevi, and Jingshown Wu, "Packet Loss Comparison of Feed-Forward and Feedback Type Asynchronous WDM Optical Routers under Self-Similar Traffic," Submitted to IEEE Commun. Letters., 2003.

[11] A. Kushwaha, S. K. Bose, Y. N. Singh, "Analytical Modeling for Performance Studies of an FLBM-Based All-Optical Packet Switch", IEEE Communication Letters, vol. 5, no. 4, pp. 227-229, Apr. 2001.

[12] Tsybakov, B. and Georganas, N.D., "On self-similar traffic in ATM queues: definitions, overflow probability bound, ard cell delay distribution", IEEE/ACM Transactions on Networking, vol. 5, issue 3, pp. 397. 409, June 1997.

[13] Attila Vidacs, Self-Similar Traffic Modeling Techniques in ATM Networks, master thesis, Technical University of Budapest, 1996.

[14] Jenny J. He, Dimitra Simeonidou and Sabih Chaudrry, "Contention resolution in optical packet-switching networks: under long-range dependent traffic," in Proc. OFC '2000, Baltimore, March 2001, paper ThU4-1.

[15] Gerardo Castanon, Lubo Tancevski and Lakshnnan Tamil, "Optical packet switching with multiple path routing," Computer Networks., vol. 32, issue 5, pp. 635-662, May 20100 . 